Quim. Nova, Vol. 36, No. 4, 524-527, 2013

\title{
CONSTITUINTES QUÍMICOS E ATIVIDADE ANTIOXIDANTE DE Byrsonima gardneriana (Malpighiaceae)
}

\author{
Thaísa Leite Rolim, Flávia Talita de Sousa Wanderley e Emídio Vasconcelos Leitão da Cunha* \\ Laboratório de Tecnologia Farmacêutica Prof. Delby Fernandes de Medeiros, Centro de Ciências da Saúde, Universidade Federal \\ da Paraíba, CP 5009, 58051-970 João Pessoa - PB, Brasil
}

Josean Fechine Tavares

Departamento de Ciências Farmacêuticas, Centro de Ciências da Saúde, Universidade Federal da Paraíba, CP 5009, 58051-970

João Pessoa - PB, Brasil

Adriana Maria Fernandes de Oliveira

Unidade Acadêmica de Ciências da Vida, Universidade Federal de Campina Grande, 58900-000 Cajazeiras - PB, Brasil

Temilce Simões de Assis

Departamento de Fisiologia e Patologia, Centro de Ciências da Saúde, Universidade Federal da Paraíba, CP 5009, 58051-970

João Pessoa - PB, Brasil

Recebido em 11/7/12; aceito em 30/10/12; publicado na web em 28/2/13

\begin{abstract}
CHEMICAL CONSTITUENTS AND ANTIOXIDANT ACTIVITY OF Byrsonima gardneriana (Malpighiaceae). The phytochemical investigation of Byrsonima gardneriana led to the isolation of five triterpenes and one flavonoid: D:B-Friedoolean5-en-3-one (1), friedoolean-14-en-3-one (2), friedelan-3-one (3), lup-20(29)-en-3-ol (4), 33-hydroxiolean-12-ene (5) and 3,3',4',5,7-pentahydroxyflavan (6). Their structures were assigned based on spectroscopic analyses, including two-dimensional NMR techniques and comparison with published spectral data. Antioxidant activities of ethanol extract and phases were measured using the 1,2-diphenyl- 2-picryl-hydrazyl (DPPH) free radical scavenging assay, evaluation of total phenolic content and trolox equivalent antioxidant capacity (TEAC).
\end{abstract}

Keywords: Byrsonima gardneriana; triterpenes; antioxidant activity.

\section{INTRODUÇÃO}

Malpighiaceae é uma família constituída por ervas, arbustos, árvores ou lianas que compreendem cerca de 75 gêneros, com

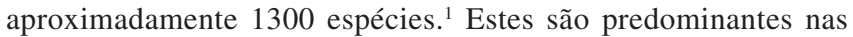
regiões tropicais e subtropicais, especialmente no continente americano. ${ }^{2,3}$ No Brasil ocorrem 45 gêneros e aproximadamente 300 espécies. ${ }^{1}$ Plantas pertencentes à família Malpighiaceae são ricas em substâncias antioxidantes como os taninos e flavonoides. ${ }^{4}$ Estudos realizados a partir do extrato clorofórmico de Byrsonima microphylla A. Juss levaram ao isolamento de duas naftoquinonas, que pela primeira vez foram relatadas em Malpighiaceae, além de flavanol e triterpenos (lupenona, $\beta$-amirina, betulina e lupeol). ${ }^{5}$ Há ainda relatos de estudos que evidenciaram a composição química do óleo floral de Byrsonima intermedia. Este óleo floral é rico em ácidos graxos livres e o principal constituinte identificado foi o ácido birsônico. ${ }^{6}$

O gênero Byrsonima é composto por cerca de 150 espécies e encontrado principalmente a partir do México, difundindo-se por toda América do Sul. ${ }^{5}$ No Brasil são encontradas no norte, nordeste e região central, podendo também ser encontradas em regiões serranas do Sudeste. São empregadas não apenas na medicina tradicional, mas também no preparo de alimentos como sucos, geleias e licores, principalmente nas regiões norte e nordeste do país. ${ }^{7}$ Espécies deste gênero são comumente empregadas pela medicina tradicional como antiasmáticas, antitérmicas e no tratamento de infecções da pele. ${ }^{7,8}$ No Nordeste brasileiro, ocorrem diversas espécies do gênero Byrsonima, havendo na literatura relatos que reportam a presença de triterpenos em Byrsonima verbascifolia e Byrsonima microphylla, e flavonoides e esteroides em Byrsonima variabilis. ${ }^{9}$ Vários

*e-mail: emidio@ltf.ufpb.br estudos relatam a química de Byrsonima crassifolia, da qual foram isolados compostos voláteis dos frutos, glicolipídeos, triterpenos, ácidos triterpênicos, catequinas e flavonoides das folhas ${ }^{10,11}$ e do tronco, proantocianidinas e taninos. ${ }^{12}$ Alguns estudos relatam ainda o isolamento de uma mistura de ácido gálico e galato de metila, quercetina-3- $O-\alpha-\mathrm{L}$-piranosídeo e a mistura de quercetina-3- $O-\beta-\mathrm{D}$ glucopiranosídeo e quercetina-3- $O-\beta$-D-galactopiranosídeo de Byrsonima crassa. ${ }^{7}$ A investigação química de outras espécies de Byrsonima relata o isolamento de esteroides, triterpenos, flavonoides e proantocianidinas. ${ }^{7,13-16}$

Os radicais livres, na forma de espécies reativas de oxigênio (EROs) e espécies reativas de nitrogênio (ERNs), são uma parte integrante da fisiologia normal. ${ }^{17}$ No entanto, as EROs exercem um papel importante nos processos degenerativos ou patológicos em várias doenças humanas, como o envelhecimento, ${ }^{18}$ câncer, doenças coronarianas, doença de Alzheimer, ${ }^{19}$ desordens neurodegenerativas, aterosclerose e inflamação. ${ }^{20}$ Substâncias antioxidantes em sistemas biológicos têm múltiplas funções, que incluem proteção contra danos oxidativos e prevenção dos danos causados pelas EROs. ${ }^{21}$ As plantas podem ser uma fonte de ampla variedade de moléculas sequestradoras de radicais livres, como compostos fenólicos (ex.: flavonoides, cumarinas, quinonas etc.), compostos nitrogenados (alcaloides), terpenoides e alguns outros metabólitos..$^{22,23}$

A literatura reporta atividade antioxidante ao extrato metanólico de Byrsonima gardneriana A. Juss; ${ }^{16}$ no entanto, não há relatos sobre o teor de fenólicos totais e atividade antioxidante do extrato etanólico bruto e das fases desta espécie. ${ }^{17} \mathrm{~A}$ referida espécie não apresenta estudos sobre seus constituintes químicos descritos na literatura, destacando-se a importância de um estudo fitoquímico criterioso da mesma. Neste artigo reportamos o isolamento e a caracterização estrutural de cinco triterpenos, 3-oxo-5-ene-glutinona (1), taraxerona (2), friedelina (3), lupeol (4) e $\beta$-amirina (5), um flavonoide, 
(+)-catequina (6). A atividade antioxidante do extrato etanólico bruto e das fases hexânica, clorofórmica, acetato de etila e n-butanólica também foi avaliada.

\section{PARTE EXPERIMENTAL}

\section{Procedimentos experimentais gerais}

Os espectros de RMN foram obtidos em espectrômetro MercuryVarian a $200\left[200 \mathrm{MHz}\left({ }^{1} \mathrm{H}\right)\right.$ e $\left.50 \mathrm{MHz}\left({ }^{13} \mathrm{C}\right)\right]$ e $500\left[500 \mathrm{MHz}\left({ }^{1} \mathrm{H}\right)\right.$ e $\left.125 \mathrm{MHz}\left({ }^{13} \mathrm{C}\right)\right]$. O solvente empregado foi $\mathrm{CDCl}_{3}$, cujos picos característicos em $\mathrm{RMN}{ }^{1} \mathrm{H}$ e ${ }^{13} \mathrm{C}$ serviram para ajuste de escala de frequência. Para as cromatografias em coluna utilizou-se como fase estacionária sílica gel 60 (70-230 mesh) da Merck. A cromatografia de camada delgada comparativa (CCDC) foi empregada para análise e reunião das frações obtidas por cromatografia em coluna. Foram utilizadas placas de vidro cuja fase fixa foi preparada com uma suspensão de sílica gel PF254 7749 (Merck) em água. As substâncias em análise foram evidenciadas pelo uso de radiação ultravioleta nos comprimentos de onda de 254 e $366 \mathrm{~nm}$ e pela impregnação das placas pelo reagente de anisaldeído sulfúrico. A rotação específica da substância 6 foi realizada no polarímetro do modelo Polarimeter, série P-2000, marca Jasco.

\section{Material vegetal}

O material vegetal, partes aéreas, de Byrsonima gardneriana, foi coletado no município de Serra Branca, Estado da Paraíba, em março de 2007. O material botânico foi identificado pela botânica Prof ${ }^{a}$ Dra. M. de F. Agra, do LTF/UFPB; uma exsicata encontra-se catalogada no Herbário Prof. Lauro Pires Xavier (JPB), da Universidade Federal da Paraíba, sob o registro de coleta AGRA 947.

\section{Procedimento para obtenção dos extratos e isolamento dos constituintes}

As partes aéreas de Byrsonima gardneriana A. Juss foram secas em estufa com ar circulante à temperatura média de $45^{\circ} \mathrm{C}$, durante 3 a 4 dias. Após a secagem, o material vegetal foi submetido a um processo de pulverização em moinho mecânico tipo Harley, obtendo-se $4 \mathrm{~kg}$ de pó seco. O material vegetal seco e pulverizado foi submetido à maceração com etanol a 95\%, durante 72 h. Após extração, a solução extrativa foi concentrada em rotavapor sob pressão reduzida à temperatura média de $45^{\circ} \mathrm{C}$, obtendo-se o extrato etanólico bruto denominado EEB $(732,0 \mathrm{~g})$.

Uma parte do EEB (350 g) foi particionado com hexano, clorofórmio, acetato de etila e n-butanol. As soluções obtidas foram tratadas com sulfato de sódio anidro $\left(\mathrm{Na}_{2} \mathrm{SO}_{4}\right)$, filtradas e concentradas sob pressão reduzida, fornecendo as fases hexânica (14,84 g), clorofórmica $(3,0 \mathrm{~g})$, acetato de etila $(56,98 \mathrm{~g})$ e n-butanólica $(24,07)$. A fase clorofórmica $(3,0 \mathrm{~g})$ foi submetida à cromatografia em coluna usando-se como adsorvente $300 \mathrm{~g}$ de sílica gel 60, da Merck, empacotada em suporte de vidro e eluída com hexano, clorofórmio e metanol em gradiente crescente de polaridade e recolheram-se 257 frações de $125 \mathrm{~mL}$ cada. A fração 13 foi eluída em um sistema de solvente hexano:clorofórmio (9:1) e apresentou-se como sólido amorfo branco, que forneceu a substância 1 (19,0 mg). As frações 25-27 e 28 foram eluídas em um sistema de solvente hexano:clorofórmio (8:2) e apresentaram-se como sólidos amorfos brancos, que forneceram as substâncias 2 (10,0 mg) e 3 (23,0 mg). A fração 58 foi eluída em um sistema de solvente hexano:clorofórmio (7:3) e apresentou-se como sólido amorfo branco, que forneceu a substância $\mathbf{5}$ (8,6 mg). A fração 45-57 foi eluída em um sistema de solvente hexano:clorofórmio (8:2) e apresentou-se como sólido branco cristalino que, após sucessivas recristalizações com acetona, forneceu a substância 4 (2,0 mg). A fase acetato de etila foi submetida à cromatografia em coluna com 200 g de sílica gel 60 da Merck empacotada em suporte de vidro e eluída com hexano, acetato de etila e metanol em gradiente crescente de polaridade e recolheram-se 60 frações de $125 \mathrm{~mL}$ cada. A fração 15-21 foi eluída em um sistema de solvente hexano:acetato (2:8), apresentou-se como pó amarelo que, após sucessivas recristalizações com acetona, forneceu a substância $\mathbf{6}(2,0 \mathrm{mg})$. Todas as substâncias faram submetidas à análise de ressonância magnética nuclear (RMN) de ${ }^{1} \mathrm{He} \mathrm{e}^{13} \mathrm{C}$.

\section{Avaliação da atividade antioxidante do extrato e das fases hexânica, clorofórmica, acetato de etila e n-butanólica}

\section{Teor de fenólicos totais}

A determinação do teor de fenólicos totais presentes nos extratos EtOH bruto e nas fases hexânica, clorofórmica, acetato de etila e n-butanólica de Byrsonima gardneriana foi realizada utilizando-se o reagente de Folin-Ciocalteau, com o ácido gálico como o composto fenólico padrão.

Alíquotas de $200 \mu \mathrm{L}$ das fases hexânica, clorofórmica, acetato de etila e n-butanólica, provenientes de uma solução estoque de $1 \mathrm{mg} / \mathrm{mL}$, solubilizadas em EtOH foram transferidas para um balão volumétrico de $5 \mathrm{~mL}$, adicionando-se $100 \mu \mathrm{L}$ do reagente de Folin-Ciocalteu e 3 $\mathrm{mL}$ de água destilada, agitando-se por $1 \mathrm{~min}$. Em seguida, $300 \mu \mathrm{L}$ de $\mathrm{Na}_{2} \mathrm{CO}_{3}(15 \%)$ foram acrescentados à mistura e agitados por $30 \mathrm{~s}$. Finalmente, a solução teve seu volume aferido para $5 \mathrm{~mL}$ com água destilada. Após 2 h, a absorbância das amostras foi medida a 760 $\mathrm{nm}$ utilizando-se cubetas de vidro. A concentração dos compostos fenólicos totais foi determinada como micrograma de equivalente de ácido gálico por grama de amostra (mg EAG/g), a partir da curva de calibração construída com padrões de ácido gálico $(0,5$ a $25 \mu \mathrm{g} / \mathrm{mL})$ considerando-se o erro padrão da média (E.P.M.).

\section{Atividade sequestradora do radical DPPH}

A atividade sequestradora de radical livre foi medida usando-se o radical DPPH, no qual a taxa de clareamento é monitorada por um comprimento de onda característico $(517 \mathrm{~nm})$, na presença da amostra. $\mathrm{Na}$ forma de radical, o DPPH absorve a $517 \mathrm{~nm}$, mas sob redução pelo antioxidante ou espécies radicalares sua absorção diminui.

Em um tubo de ensaio com $5 \mathrm{~mL}$ de uma solução de DPPH (23,6 $\mu \mathrm{g} / \mathrm{mL}$ em EtOH) foram misturadas quantidades apropriadas do extrato e das fases de Byrsonima gardneriana (provenientes de uma solução estoque de $1 \mathrm{mg} / \mathrm{mL}$ e obtidas através de uma triagem preliminar), acompanhadas pela homogeneização. Após 30 min de agitação em aparelho de ultrassom, a quantificação do radical DPPH foi realizada em espectrofotômetro UV-visível a $517 \mathrm{~nm}$. As análises foram realizadas em triplicata $(n=3)$. A percentagem da atividade sequestradora (\% AS) foi calculada pela equação:

$$
\% \text { AS }=100 \times(\text { Acontrole }- \text { Aamostra }) / \text { Acontrole }
$$

onde Acontrole é a absorbância do controle, uma solução que contém apenas o radical DPPH e EtOH, e Aamostra é a absorbância do radical na presença dos extratos ou do padrão ácido ascórbico.

As medidas foram calculadas e os resultados expressos como média \pm E.P.M. A eficiência antirradicalar foi estabelecida usando-se análise de regressão linear no intervalo de confiança de $95 \%(\mathrm{p}<0,05)$ obtido pelo programa de estatística GraphPad Prism 4.0. Os resultados estão representados pelo valor da $\mathrm{CE}_{50}$, que representa a concentração da amostra necessária para sequestrar 50\% dos radicais DPPH. Os extratos e substâncias são considerados ativos quando apresentam 
$\mathrm{CE}_{50}<500 \mu \mathrm{g} / \mathrm{mL} .{ }^{24}$ Como padrão de referência foi utilizado o ácido ascórbico.

Capacidade antioxidante equivalente ao trolox (CAET)

A determinação da CAET foi realizada, usando o Trolox (ácido 6-hidróxi-2,5,7,8-tetrametilcromano-2-carboxílico) como composto padrão. ${ }^{25}$

O cátion radical do ácido 2,2'-azinobis-(3-etilbenzotiazolina-6-sulfônico) (ABTS) foi preparado pela reação de $7 \mathrm{mM}$ de ABTS com 2,45 mM de persulfato de potássio. A mistura foi deixada no escuro, à temperatura ambiente, por 12-16 h, antes do uso. Para a avaliação da atividade a solução ABTS foi diluída em etanol (1:90 v/v, aproximadamente) até se obter uma absorbância de 0,7 $\pm 0,02$, no comprimento de onda de $734 \mathrm{~nm}$. Quantidades apropriadas da fase clorofórmica de Byrsonima gardneriana (provenientes de uma solução estoque de $1 \mathrm{mg} / \mathrm{mL}$ e obtidas através de uma triagem preliminar) ou do padrão Trolox (concentrações finais de 6 a $18 \mu \mathrm{M}$ ) foram misturadas com $5 \mathrm{~mL}$ da solução de ABTS. As soluções foram agitadas e, após 10 min, a absorbância das amostras e do padrão foram medidas, utilizando-se cubetas de vidro. As análises foram realizadas em triplicata e a percentagem de inibição (\% I) em relação ao branco foi calculada pela equação:

$$
\% \mathrm{I}=100 \times(\text { Abranco }- \text { Aamostra }) / \text { Abranco }
$$

\section{RESULTADOS E DISCUSSÃO}

A partição líquido-líquido do extrato etanólico das partes aéreas de Byrsonima gardneriana A. Juss, seguida por cromatografia em coluna com sílica gel da fase clorofórmica, levou ao isolamento de cinco triterpenos. Após análise dos dados de $\mathrm{RMN}{ }^{1} \mathrm{H}$ e ${ }^{13} \mathrm{C}$ e uni e bidimensionais, os quais nos possibilitaram evidenciar as correlações entre os hidrogênios e seus respectivos carbonos e confirmar as posições dos grupos metílicos, e a comparação desses valores com os da literatura, foi possível identificar as referidas substâncias como sendo: (1) o triterpeno D:B-friedo-oleanano-5-ene-3-ona; ${ }^{26}$ (2) o triterpeno da série taraxarano, friedo-olean-14-en-3-ona, ${ }^{26}$ este composto é usualmente denominado de taraxerona, ambas as substâncias, 1 e 2, foram isoladas pela primeira vez no gênero em estudo; (3) o triterpeno da série friedelano, friedelan-3-ona, ${ }^{27}$ também conhecido como friedelina; (4) o triterpeno da série lupano, lup-20(29)-en-3-ol ${ }^{28}$ também denominado lupeol; (5) o triterpeno da série oleanano, $3 \beta$-hidroxiolean-12-eno, ${ }^{29}$ o qual é usualmente denominado de $\beta$-amirina. As substâncias 3, 4 e $\mathbf{5}$ foram isoladas pela primeira vez na espécie em estudo.

Separações cromatográficas com sílica gel da fase acetato de etila conduziram à obtenção de um flavonoide (6) o 3,3',4',5,7- penta-hidroxiflavana. ${ }^{30,31} \mathrm{O}$ valor do $[\alpha]_{\mathrm{D}}^{20}+3,0$ (c. 0,001, acetona) confirma a estereoquímica da substância como sendo a (+)-catequina, sendo esta isolada pela primeira vez na espécie em estudo (Figura 1).

\section{Atividade sequestradora do radical DPPH}

O radical estável DPPH tem sido extensivamente usado para a determinação da atividade antioxidante, que é a atividade sequestradora de compostos antioxidantes puros, extratos de plantas, frutos e alimentos. ${ }^{32}$

A atividade antioxidante do EEB e das fases hexânica, clorofórmica, acetato de etila e n-butanólica de Byrsonima gardneriana, medida pela atividade sequestradora do radical DPPH, está expressa na Tabela 1.

A fase AcOEt apresentou a melhor atividade antioxidante $(3,45 \pm 0,043 \mathrm{mg} / \mathrm{mL})$, semelhante ao valor do ácido ascórbico

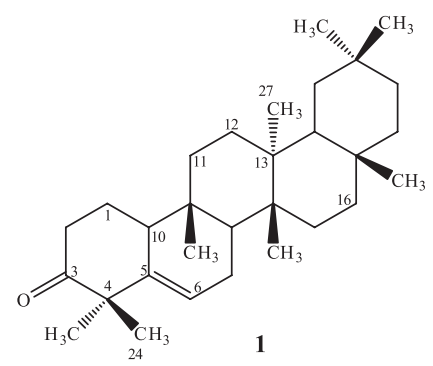

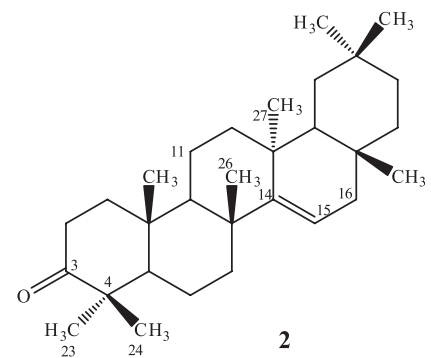<smiles>C[C@H]1C(=O)CCC2[C@]3(C)CC[C@]4(C)C5CC(C)(C)CC[C@]5(C)CC[C@]4(C)C3CC[C@]21C</smiles>

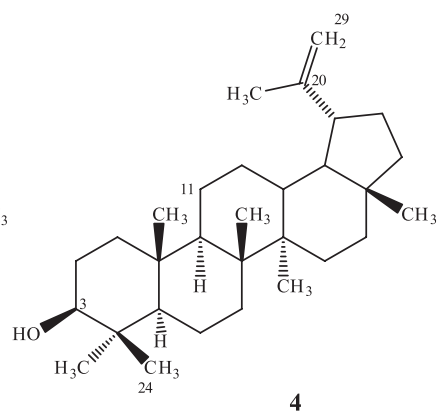

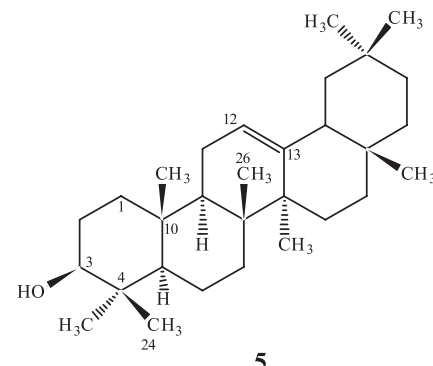<smiles>Oc1cc(O)c2c(c1)OC(c1ccc(O)c(O)c1)C(O)C2</smiles>

Figura 1. Estruturas dos constituintes químicos isolados de Byrsonima gardneriana

Tabela 1. Atividade sequestradora do radical livre DPPH, das fases e do extrato bruto de Byrsonima gardneriana

\begin{tabular}{cc}
\hline Amostras & DDPH $\left(\mathrm{CE}_{50}\right)$ \\
\hline Ácido ascórbico & $3,12 \pm 0,023$ \\
EEB & $4,955 \pm 0,079$ \\
$\mathrm{HEX}$ & $24,283 \pm 0,252$ \\
$\mathrm{CHCl}_{3}$ & $10,212 \pm 0,016$ \\
$\mathrm{n}-\mathrm{BuOH}$ & $3,840 \pm 0,005$ \\
$\mathrm{AcOEt}$ & $3,454 \pm 0,04$ \\
\hline
\end{tabular}

$(3,12 \pm 0,023 \mathrm{mg} / \mathrm{mL})$ utilizado como padrão. A fase n-BuOH $(3,840$ $\pm 0,005 \mathrm{mg} / \mathrm{mL}$ ) apresentou atividade antioxidante semelhante à fase AcOEt; isto demonstra que as substâncias responsáveis pela atividade antioxidante estão presentes nas fases mais polares.

\section{Avaliação do teor de fenólicos totais}

O ensaio de Folin-Ciocalteu é um método rápido e simples de se determinar o teor de compostos fenólicos em amostras. A fase AcOEt apresentou o maior teor de compostos fenólicos totais $(509,947 \pm$ 7,387), seguida das frações n-BuOH $(492,261 \pm 4,904 \mathrm{mEAG} / \mathrm{g})$, $\operatorname{EEB}(425,105 \pm 8,523 \mathrm{mEAG} / \mathrm{g}), \mathrm{CHCl}_{3}(243,29 \pm 3,897 \mathrm{mEAG} / \mathrm{g})$, $\operatorname{HEX}(80,42 \pm 0,693 \mathrm{mEAG} / \mathrm{g})$ (Tabela 2$)$.

Compostos fenólicos, como flavonoides, encontrados em plantas possuem muitas atividades biológicas, incluindo potente poder antioxidante, contribuindo para as defesas do organismo. ${ }^{33} \mathrm{~A}$ bioatividade dos fenólicos pode ser atribuída a sua habilidade para quelar metais, inibir a lipoxigenase e sequestrar radicais livres. ${ }^{34}$ 
Tabela 2. Teor de fenólicos totais das fases e do extrato bruto de Byrsonima gardneriana

\begin{tabular}{cc}
\hline Amostras & Teor de fenólicos totais (mEAG/g) \\
\hline EEB & $425,105 \pm 8,523$ \\
$\mathrm{HEX}$ & $80,42 \pm 0,693$ \\
$\mathrm{CHCl}_{3}$ & $243,29 \pm 3,897$ \\
$\mathrm{n}-\mathrm{BuOH}$ & $509,947 \pm 7,387$ \\
$\mathrm{AcOEt}$ & $492,261 \pm 4,904$ \\
\hline
\end{tabular}

\section{Capacidade antioxidante equivalente ao Trolox (CAET)}

A geração do cátion radical ABTS forma a base de um método espectrofotométrico que tem sido aplicado para a medida da atividade antioxidante de soluções de substâncias puras, misturas aquosas e bebidas. ${ }^{35}$

A Tabela 3 expressa os valores da $\mathrm{CE}_{50}$ do extrato e das fases hexânica, clorofórmica, acetato de etila e n-butanólica de Byrsonima gardneriana. A fase $\mathrm{n}-\mathrm{BuOH}(3,15 \pm 0,055 \mathrm{mg} / \mathrm{mL})$ apresentou a melhor atividade antioxidante neste teste, semelhante ao padrão Trolox $(3,02 \pm 0,014 \mathrm{mg} / \mathrm{mL})$, seguida pelas fases AcOEt $(3,453 \pm$ $0,101 \mathrm{mg} / \mathrm{mL}), \operatorname{EEB}(3,833 \pm 0,088 \mathrm{mg} / \mathrm{mL}), \mathrm{CHCl}_{3}(8,466 \pm 0,063$ $\mathrm{mg} / \mathrm{mL}), \operatorname{HEX}(20,68 \pm 0,372 \mathrm{mg} / \mathrm{mL})$.

Tabela 3. Capacidade antioxidante equivalente ao Trolox (CAET), das fases e do extrato bruto de Byrsonima gardneriana

\begin{tabular}{cc}
\hline Amostras & ABTS $\left(\mathrm{CE}_{50-} \mathrm{mg} / \mathrm{mL}\right)$ \\
\hline Trolox & $3,02 \pm 0,014$ \\
EEB & $3,833 \pm 0,088$ \\
$\mathrm{HEX}$ & $20,68 \pm 0,372$ \\
$\mathrm{CHCl}_{3}$ & $8,466 \pm 0,063$ \\
$\mathrm{n}-\mathrm{BuOH}$ & $3,15 \pm 0,055$ \\
$\mathrm{AcOEt}$ & $3,453 \pm 0,101$ \\
\hline
\end{tabular}

Assim como ocorreu no teste da atividade sequestradora do radical DPPH, as fases AcOEt e n-BuOH apresentaram a melhor atividade antioxidante, o que caracteriza a presença de substância com potencial atividade antioxidante.

A correlação entre a concentração de fenólicos totais e atividade antioxidante tem sido amplamente estudada em alimentos, frutos e vegetais. ${ }^{36,37}$ Polifenóis têm sido relatados como sendo os responsáveis pela atividade antioxidante dos extratos botânicos. ${ }^{33} \mathrm{~A}$ grande correlação entre o teor de fenólicos totais e a atividade antioxidante nos dois testes utilizados, atividade sequestradora do radical livre DPPH $\left(\mathrm{R}^{2}=0,89\right)$ e capacidade antioxidante equivalente ao Trolox $\left(\mathrm{R}^{2}=0,91\right)$, indica que as substâncias fenólicas, como os flavonoides que foram isolados neste estudo, são responsáveis pela boa atividade antioxidante desta planta.

\section{REFERÊNCIAS}

1. Souza, V. C.; Lorenzi, H.; Botânica sistemática: guia ilustrado para identificação das famílias de Fanerógamas nativas e exóticas no Brasil, $2^{\mathrm{a}}$ ed., Nova Odessa: São Paulo, 2008.

2. Anderson, W. R.; Memoirs of the New York Botanical Garden 1981, 32, 21.

3. Ribeiro, J. E. L. S.; Hopkins, M. J. G.; Vicentini, A.; Sothers, C. A.; Costa, M. A. S.; Brito, J. M.; Souza, M. A. D.; Martins, L .H. P.; Lohmann, L. G.; Assunção, P. A. C. L.; Pereira, E. C.; Silva, C. F.; Mesquita, M. R.; Procópio, L. C.; Floresta da reserva Ducke: guia de identificação das plantas vasculares de uma floresta de terra-firme na Amazônia Central, INPA: Manaus, 1999.
4. Gottlieb, O. R.; Borin, M. R. M. B. Em Farmacognosia: da planta ao medicamento; Simões, C. M. O., org.; 3a ed., editora da UFRGS: Porto Alegre, 2001.

5. Aguiar, R. M.; David, J. P.; David, J. M.; Phytochemistry 2005, 66, 2388.

6. Reis, M. G.; Faria, D. A.; Santos, I. A.; J. Chem. Ecol. 2007, 33, 1421.

7. Sannomiya, M.; Fonseca, V. B.; Da Silva, M.A.; Rocha, L. R. M.; Dos Santos, L. C.; Hiruma-Lima, C. A.; Souza-Brito, A. R. M.; Vilegas, W.; J. Ethnopharmacol. 2005, 97, 1.

8. Caceres, A.; Figueroa, L.; Taracena, A. M.; Samayoa, B.; J. Ethnopharmacol. 1993, 39, 77.

9. David, J. M.; Santos, F. A.; Guedes, M. L. da S.; Quim. Nova 2003, 26, 484.

10. Amarquaye, A.; Che, C. T.; Bejar, E.; Malone, M. H.; Fong, H. H. S.; Planta Med. 1994, 60, 85.

11. Rastrelli, L.; Detommasi, N.; Berger, I.; Caceres, A.; Saravia, A.; De Simone, F.; Phytochemistry 1997, 45, 647.

12. Geiss, F.; Heinrich, M.; Hunkler, D.; Rimpler, H.; Heinrich, M.; Phytochemistry 1995, 39, 635.

13. Bejar, E.; Amarquaye, A.; Che, C. T.; Malone, M. H.; Fong, H. H. S.; Int. J. Pharmacogn. 1995, 33, 25.

14. Miller, D. D. In Food Chemistry; Fennema, O. R., ed.; Marcel Dekker: New York, 1996, p. 618-649.

15. Mendes, C. C.; Cruz, F. G.; David, J. M.; Nascimento, I. P.; David, J. P.; Quim. Nova 1999, 22, 185

16. Sannomiya, M.; Rodrigues, C. M.; Coelho, R. G.; Santos, L. C.; Hiruma-Lima, C. A.; Brito, A. R. M. S.; Vilegas, W.; J. Chromatogr., A 2004, 1035, 47.

17. David, J. P.; Meira, M.; David, J. M.; Fitoterapia 2007, 78, 215.

18. Halliwell, B.; Free Radical Biol. Med. 1989, 7, 645.

19. Burns, J.; Gardner, P. T.; Matthew, D.; Duthie, C. G.; Lean, M. E.; Crozier, A.; J. Agric. Food Chem. 2001, 9, 5797.

20. Diaz, M. N.; Frei, B.; Vita, J. A.; Keaney, J. F.; N. Engl. J. Med. 1997, 337, 408.

21. Aruoma, O. I.; J. Am. Oil Chem. Soc 1998, 75, 199.

22. Kannan, R. R. R.; Arumugam, R.; Anantharaman, P.; Asian Pac. J. Trop. Med. 2010, 898.

23. Larson, R. A.; Phytochemistry 1988, 27, 969.

24. Campos, M. G.; Webby, R. F.; Markham, K. R.; Mitchell, K. A.; Da Cunha, A. P.; J. Agric. Food Chem. 2003, 51, 742.

25. Re, R.; Pellegrini, N.; Proteggente, A.; Pannala, A.; Yang, M.; RiceEvans, C.; Free Radical Biol. Med. 1999, 26, 1231.

26. Mahato, S. B.; Kundu, A. P.; Phytochemistry 1994, 37, 1517.

27. Cano, A.; Bucio, J. L.; Espinoza, M.; Cancino-Ruíz, A.; Rev. Soc. Quim. México 2000, 44, 155.

28. Burns, D.; Reynolds, W. F.; Buchanan, G.; Reese, P. B.; Enriquez, R. G.; Magn. Reson. Chem. 2000, 38, 488.

29. de Carvalho, M. G.; Velandia, J. R.; Oliveira, L. F.; Bezerra, F. B.; Quim. Nova 1998, 21, 740 .

30. Bae, Y. S.; Burger, J. F.; Steynberg, J. P.; Ferreira, D.; Hemingway, R. W.; Phytochemistry 1994, 35, 473.

31. Delazar, A.; Talischi, B.; Nazemiyeh, H.; Rezazadeh, H.; Nahar, L. S. D.; Sarker, S. D.; Rev. Bras. Farmacogn. 2006, 16, 286.

32. Wong, S. P.; Leong, L. P.; Koh, J. H. W.; Food Chem. 2006, 99, 775.

33. Soobratte, M. A.; Neergheen, V. S.; Luximon-Ramma, A.; Aruoma, O. I.; Bahorun, T.; Mutat. Res. 2005, 579, 200.

34. Mallavadhani, U. V.; Sudhakar, A. V. S.; Satyanarayana, K. V. S.; Mahapatra, A.; Li, W.; Van Breemen, R. B.; Food Chem. 2006, 95, 58.

35. Cui, C. B.; Tezuka, Y.; Kikuchi, T.; Nakano, H.; Tamaoki, T.; Park, J. H.; Chem. Pharm. Bull. 1992, 40, 889.

36. Jayaprakasha, G. K.; Girennavar, B.; Patil, B. S.; Bioresour. Technol. 2008, 99, 4484.

37. Klimczak, I.; Malecka, M.; Szlachta, M.; Gliszczynska, A.; J. Food Comp. Anal. 2007, 20, 313. 